ARTIGO ORIGINAL ORIGINAL ARTICLE

\section{Estenose valvular aórtica e o uso de TAVI: revisão narrativa das evidências publicadas e avaliação básica de custos}

\author{
Aortic valve stenosis and the use of TAVI: narrative review \\ of published evidences and basic costs assessment
}

Wilson Follador ${ }^{1}$, Carlos Fernando Mendoza², Janaina Gonçalves³, Larissa Sartori ${ }^{3}$, Cristina Nunes Ferreira ${ }^{3}$

DOI: $10.21115 /$ JBES.v10.n1.p36-44

\begin{abstract}
Palavras-chave:
implante transcateter de válvula aórtica, TAVI, estenose aórtica, economia da saúde
\end{abstract}

\begin{abstract}
RESUMO
Objetivo: Revisar as evidências clínicas da estenose valvular aórtica em suas dimensões médica e econômica, analisando as alternativas de tratamento cirúrgico em pacientes de alto risco cirúrgico com foco na tecnologia do implante transcateter de válvula aórtica (TAVI). Métodos: Foi elaborada uma revisão narrativa das publicações relacionadas à estenose vascular aórtica e às metodologias de tratamento cirúrgico, assim como a comparação entre a metodologia TAVI e as abordagens convencionais (substituição cirúrgica de válvula aórtica por esternotomia, valvuloplastia por balão intra-aórtico e tratamento farmacológico), abordando ainda dados sobre algumas diferentes marcas e gerações de dispositivos de TAVI oferecidas no mercado brasileiro, avaliando a eficácia, a segurança e o custo de tratamento. Resultados: Em pacientes portadores de estenose aórtica grave sintomática e inoperáveis pela abordagem convencional, o uso do TAVI mostrou ser uma alternativa mais segura, eficaz e econômica em comparação aos tratamentos convencionais. Estudos com robustez metodológica mostram que esse procedimento alcança taxas superiores a $80 \%$ de sobrevida em um ano e aproximadamente 30\% em cinco anos, mesmo em pacientes de alto risco. No entanto, foram observadas diferenças significativas nos desfechos entre diferentes estudos, que podem ser atribuídas não apenas ao desenho e à amostragem, mas às diferenças entre as diversas opções de gerações e marcas de dispositivos para TAVI disponíveis no mercado. Essas opções diferem principalmente na estrutura, via de inserção e modo de expansão. As válvulas de terceira geração vêm apresentando os melhores resultados em termos de eficácia, segurança e facilidade no procedimento, que resultam em mais benefícios aos pacientes e equilibram os custos de tratamento em médio e longo prazos. Conclusões: Estudos com evidências robustas avaliando desfechos representativos para o tratamento da estenose aórtica grave e sintomática mostram que a tecnologia minimamente invasiva TAVI é eficaz, segura no tratamento de pacientes de alto risco ou inoperáveis, com melhorias significativas nos dispositivos de terceira geração.
\end{abstract}

\section{Keywords:}

transcatheter aortic valve implantation, TAVI, aortic stenosis, health economic

\begin{abstract}
Objective: To review the clinical evidences of aortic valve stenosis in its medical and economical settings, reviewing the surgical treatment alternatives in patients at high surgical risk focused on the transcatheter aortic valve implantation (TAVI) technology. Methods: A structured review of the publications related to the disease and to the surgical treatment methodologies was conducted, followed by a search for information about the TAVI methodology, with comparisons between this method and conventional approaches (surgical aortic valve replacement by means of sternotomy, valvuloplasty via intra-aortic balloon and pharmacological treatment) and comparisons between different brands and generations of TAVI devices, evaluating the treatment efficacy, safety and cost.
\end{abstract}

\footnotetext{
Recebido em: 12/03/2018. Aprovado para publicação em: 02/04/2018.

1. TechValue Consultoria, São Paulo, SP, Brasil.

2. Edwards Lifesciences, Cidade do México, México.

3. Edwards Lifesciences, São Paulo, SP, Brasil.

Instituição onde o trabalho foi realizado: TechValue.

Financiamento: Este estudo foi financiado pela Edwards Lifesciences.

Autor correspondente: Cristina Nunes Ferreira. Rua Verbo Divino, 1547, $1{ }^{\circ}$ andar, São Paulo, SP. CEP: 04719-000.

E-mail: cristina_nunes@edwards.com
} 
Results: In patients with severe symptomatic aortic stenosis who cannot be operated using the conventional approach, the use of TAVI was shown to be a safer, more effective and more economical alternative compared to the conventional treatments. The studies of the best methodology show that this is a safe, effective and curative procedure, reaching rates above $80 \%$ in 1-year survival and of approximately $30 \%$ in 5-year survival, even in patients at high risk. However, significant differences in the outcomes among different studies were observed, which may be attributed to the design and sampling as well as to the differences among the several options of TAVI devices available on the market. These options differ in structure, insertion route, expansion mode and in the several generations of valves. Third-generation valves have been presenting the best results in terms of efficacy, safety and procedure easiness, which result in more benefits to patients and balance the medium-term and long-term treatment cost. Conclusions: The most robust studies evaluating the TAVI valves show that this minimally invasive technology is effective and safe for the treatment of severe symptomatic aortic stenosis in patients at high risk or who cannot be operated.

\section{Introdução}

A estenose aórtica (EAo) é a redução da luz da válvula aórtica causada pela menor flexibilidade de seus folhetos, bem como por lesão, inflamação e calcificação ou espessamento do tecido, que acarreta a obstrução parcial do fluxo sanguíneo do ventrículo esquerdo para a aorta. As principais etiologias da EAo são calcificação valvar, febre reumática, infecções e malformações congênitas (Nishimura et al., 2014; Tarasoutchi et al., 2011).

O desenvolvimento natural da EAo em adultos comumente é lento e insidioso, com um período de latência de 10 a 20 anos (Ren \& Lange, 2017). A dispneia ao esforço é, com frequência, a queixa inicial. Outros sintomas inespecíficos que se desenvolvem precocemente são fadiga, angina, vertigem e síncope, que podem não ser patognomônicos em indivíduos com baixa atividade física (Ren \& Lange, 2017; Schwartz \& Zipes, 2015).

Após o início dos sintomas da doença, geralmente os pacientes com EAo sofrem piora importante de seu prognóstico, com sobrevida média de dois a três anos (Figura 1) e aumento significativo do risco de morte súbita (Otto \& Bonow, 2014; Thaden et al., 2014). A sobrevida pode ser ainda menor na coexistência de comorbidades cardíacas. Em pacientes cujas condições médicas impedem uma abordagem cirúrgica, a taxa de sobrevida é pequena, de aproximadamente 55\% em um ano e de 3\% em cinco anos (Brown et al., 2008) (Figura 1).

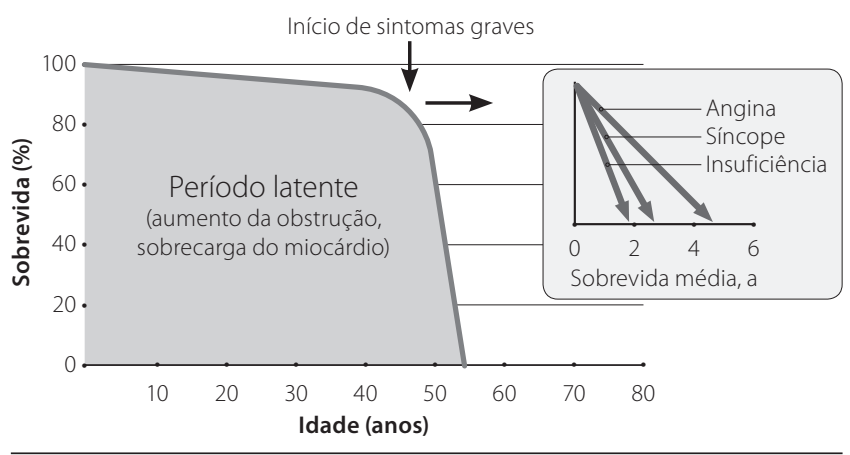

Figura 1. Curso da doença na estenose de válvula aórtica.
Uma metanálise realizada por Osnabrugge et al. (2013) relatou que a prevalência populacional de EAo variou de 2,6\% a 22,8\% entre diferentes estudos. Em indivíduos com idade superior a 75 anos, a prevalência de EAo foi de 12,4\% (IC 95\% 6,6\%-18,2\%), e as formas graves de EAo mostraram prevalência de 3,4\% (IC 95\% 1,1\%-5,7\%), com alta heterogeneidade entre os estudos avaliados na metanálise (Osnabrugge et al., 2013). Valores aproximados foram apresentados por Schwartz \& Zipes (2015), para os quais as prevalências da EAo em pessoas com idade superior a 65 anos são de 9\% na doença leve, $5 \%$ na doença moderada e $2 \%$ na doença grave (Schwartz \& Zipes, 2015).

Nos EUA, a EAo é uma importante causa de morbidade e mortalidade entre os idosos, causando mais de 13.000 óbitos anuais, e aumento na mortalidade em que a EAo está presente, mesmo não sendo a causa direta de morte (Hussain et al., 2016).

Pacientes com EAo grave são geralmente enquadrados nas classes funcionais III ou IV da NYHA' (incapazes de empreender qualquer esforço físico, em muitos casos tendo a vida restrita ao leito), apresentando cardiomegalia, insuficiência cardíaca congestiva e comprometimento severo das habilidades físicas.

\section{Custo da doença}

Ainda que a EAo se manifeste nos anos mais tardios de vida de um portador e que a perspectiva de sobrevida seja curta, as manifestações e complicações tipicamente associadas podem implicar custos elevados. Clark et al. (2012) conduziram um estudo de cinco anos que avaliou desfechos clínicos, consumo de recursos médicos e custos advindos do tratamento de 2.150 indivíduos portadores de EAo grave, sem substituição de válvula aórtica, por meio de análise retrospectiva de dados obtidos pelo sistema Medicare. Durante o período analisado, foi observado que a taxa global de mortalidade foi

1 New York Heart Association (vide http://www.heart.org/HEARTORG/ Conditions/HeartFailure/AboutHeartFailure/Classes-of-Heart-Failure_ UCM_306328_Article.jsp\#.WSaS72jysdU) 
de 88,4\%, com médias de sobrevida de 1,8 ano, 1,9 internação de 11,5 dias a cada ano por paciente. Além disso, 52\% dessa população foi referida para um serviço de skilled nursing care (unidade de enfermagem especializada) e 28\% para um hospice care (unidade de cuidados paliativos). A média dos custos médicos em cinco anos foi de US\$ $63.844 \pm$ US\$ 67.181 por paciente, sendo a maior parte devida a internações hospitalares (49,7\%, US\$ $31.714 \pm$ US\$ 35.129), serviços médicos (16,5\%, U\$ $10.531 \pm$ US\$15.366) e cuidados de enfermagem $(10,4 \%$, US\$ $6.668 \pm$ US\$ 112.525 dólares); os custos remanescentes $(23,4 \%)$ distribuíram-se entre outros tipos de serviços de saúde (Clark et al., 2012).

\section{Tratamento}

A maioria dos pacientes com doença valvular cardíaca apresenta condição leve a moderada, mas até 5,7\% (Osnabrugge et al., 2013) apresentam a doença em condição grave e sintomática, para a qual a efetividade do tratamento conservador (fármacos e valvuloplastia por balão intra-aórtico) é baixa, exigindo tratamento cirúrgico (Otto \& Bonow, 2014). A cirurgia convencional - substituição cirúrgica da válvula aórtica por acesso por meio de esternotomia, ou AVR (aortic valve replacement) - vinha há 50 anos sendo o padrão-ouro para essa abordagem, com o reconhecimento de que oferece expressivo prolongamento da sobrevida, melhora dos sintomas e baixas taxas de complicações periprocedurais (Conitec, 2013; Kodali et al., 2017). Vasques et al. (2012) realizaram uma extensa metanálise de 48 estudos, englobando 13.216 pacientes de idade superior a 80 anos submetidos à $A V R$, a qual mostrou que as taxas de sobrevida em 1, 5 e 10 anos após a intervenção foram de $87,6 \%, 65,4 \%$ e $29,7 \%$, respectivamente (Vasques et al., 2012). A Figura 2 apresenta, de forma esquemática, a evolução da EAo e as alternativas disponíveis a cada etapa.

No entanto, a realização da AVR apresenta riscos, os quais se apresentam particularmente mais elevados em pacientes com idade avançada e os portadores de comorbidades tais como cirurgia cardíaca prévia, doença pulmonar

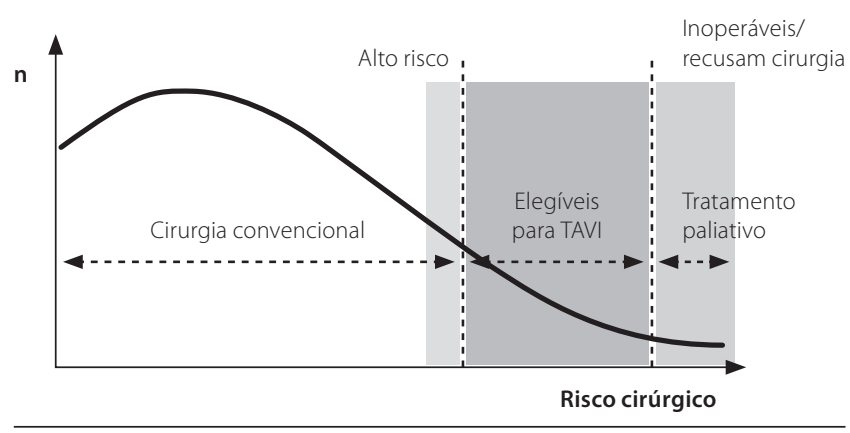

Figura 2. Mapa conceitual do continuum das abordagens cirúrgicas na EAo grave. crônica, doença vascular periférica, AVC, insuficiência renal, doença arterial coronariana e fragilidade física. Além desses, existem outros fatores que aumentam os riscos da AVR, tais como a "aorta em porcelana" (extensa calcificação da aorta ascendente e/ou do arco aórtico) e deformidades da parede torácica (Tarasoutchi et al., 2011). Uma revisão sistemática conduzida por Amato et al. (2016) mostrou que 5,7\% dos pacientes de alto risco submetidos a AVR morrem durante o procedimento, enquanto em 30 dias após a cirurgia 4\% são acometidos por AVC e 23,8\% apresentam grandes sangramentos (Amato et al., 2016). Outras complicações observadas são a disfunção da prótese, vazamento paravalvular, formação de trombos, êmbolos arteriais, endocardite infecciosa e problemas associados à anticoagulação; a gravidade das complicações varia de acordo com o tipo de prótese e outras variáveis clínicas, com frequência de pelo menos 3\% (Tarasoutchi et al., 2011).

Estima-se que a AVR não seja um procedimento aplicável em pelo menos um terço dos pacientes com EAo sintomática grave, os quais mostram risco cirúrgico maior do que o benefício esperado; esse risco é avaliado com o apoio de modelos de predição tais como o EuroSCORE logístico, o EuroSCORE II e o escore da Society of Thoracic Surgeons (STS) (Conitec, 2013; Kodali et al., 2017; Nashef et al., 1999, 2002; Shroyer et al., 2003).

Para sobrepujar tais dificuldades, foi desenvolvido o método TAVI (transcatheter aortic valve implantation - implante de transcateter de válvula aórtica), que foi usado pela primeira vez em 2002 e vem representando uma alternativa eficaz e segura no tratamento cirúrgico de pacientes com EAo grave e com restrições ao procedimento por AVR (Kodali et al., 2017; Leon et al., 2010; Otto \& Bonow, 2014). Esse procedimento também pode ser denominado TAVR (transcatheter aortic valve replacement). A técnica TAVI envolve o transporte de uma válvula aórtica artificial através de um cateter que é introduzido por uma grande artéria periférica (ou por via transapical) e que, ao ser posicionado no sítio da válvula natural doente, libera e expande o dispositivo artificial, o qual passa a desempenhar as funções que estavam prejudicadas pela EAo.

TAVI é um procedimento minimamente invasivo indicado no tratamento cirúrgico de pacientes com EAo grave e sintomática que são considerados de alto risco ou inoperáveis por AVR. Esse método se tornou o tratamento padrão-ouro para os portadores de EAo grave inviáveis para a AVR (Wendler et al., 2017), com múltiplos estudos documentando uma ampla gama de desfechos favoráveis, tais como aumento de sobrevida, melhora da sintomatologia e ganhos de qualidade de vida, assim como a redução do número de reospitalizações, entre outros benefícios (Otto et al., 2017).

Algumas grandes sociedades médicas mundiais já emitiram diretrizes que recomendam o uso de TAVI no tratamento da EAo, destacando-se: AHA/ACC Guideline for the Manage- 
ment of Patients With Valvular Heart Disease (2014)²; ESC Guidelines for the Diagnosis and Treatment of Acute and Chronic Heart Failure (2016) $)^{3}$ e o National Institute for Health and Care Excellence (NICE, em 2012) ${ }^{4}$.

O custo mais elevado da TAVI em relação à AVR tem sido um obstáculo para o seu amplo uso, muito embora haja demonstrações de que esse custo é diluído ao longo do tempo (Arnold et al., 2014) e de que o impacto orçamentário é baixo, por ser elegível apenas a uma parcela reduzida dos pacientes, conforme pode ser observado na Figura 2. Isso posto, deve ser considerado o fato de que a metodologia TAVI é não apenas eticamente necessária, por envolver uma necessidade médica que não é atendida por outra alternativa que proporcione iguais desfechos (conforme será demonstrado), mas também economicamente viável.

\section{TAVI versus AVR ou tratamento convencional}

A eficácia e a segurança do TAVI em relação à AVR têm sido determinadas mediante estudos de efetividade comparada, utilizando a técnica de metanálise. O principal objetivo deste capítulo é determinar o tamanho do efeito clínico e econômico da TAVI vs. a AVR, além da vantagem da metodologia quando aplicada a pacientes para os quais a AVR mostra um risco superior ao benefício.

Siontis et al. (2016) desenvolveram uma metanálise de ensaios clínicos randomizados que compara o TAVI com AVR em relação à eficácia e à segurança sob diversas condições de risco em subgrupos. Foram identificados quatro ensaios elegíveis pelos critérios definidos, os quais atribuíram, de forma randômica, 1.898 pacientes para TAVI e 1.908 para AVR. A média do escore STS foi 8 para os pacientes envolvidos nos

2 Disponível em: http://circ.ahajournals.org/content/circulationaha/early/2014/02/27/CIR.0000000000000029.full.pdf

3 Disponível em: https://doi.org/10.1093/eurheartj/ehw128

4 Disponível em: https://www.nice.org.uk/guidance/ipg421 ensaios de alto risco (PARTNER $1 \mathrm{~A}^{5}$ e US CoreValve High Risk ${ }^{6}$ ) e 4 para aqueles de risco intermediário (NOTION e PARTNER $\left.2 A^{8}\right)$. O endpoint primário da metanálise foi morte por todas as causas, que mostrou redução significativa de $13 \%$ em favor de TAVI (HR=0,87; IC 95\% 0,76-0,99; $p=0,038$ ) (Figura 3). Entre os desfechos secundários, o uso de TAVI foi associado a menores taxas de insuficiência renal, de novos casos de fibrilação atrial e de sangramento, enquanto a AVR mostrou menores taxas de complicações vasculares importantes, de novos implantes de marca-passo e de regurgitação paravalvular (Siontis et al., 2016).

Uma vez demonstrado que o uso de TAVI traz benefícios estatisticamente significativos de sobrevida no tratamento cirúrgico de EAo grave em pacientes de alto risco cirúrgico, cumpre analisar a possibilidade de tratar esses pacientes com valvuloplastia por balão intra-aórtico. Tal possibilidade deve ser descartada diante das evidências de que a valvuloplastia por balão intra-aórtico (BAV) apresenta benefícios apenas sintomáticos e de curto prazo.

Dois estudos de registro de casos com grande número de pacientes envolvidos $(n=1.166)$ - o NHLBI Registry (National Heart, Lung, and Blood Institute) e o Mansfield Scientific Aortic Valvuloplasty Registry - demonstraram que a valvuloplastia por balão intra-aórtico leva a cerca de 20\% a 25\% de casos de complicações e 3\% a 4\% de morte nas primeiras 24 horas pós-procedimento, assim como $64 \%$ de reospitalizações por reestenose e mortalidade de $8 \%$ antes da alta hospitalar, por causas cardiovasculares (Kodali et al., 2017). Kapadia et al. (2015), analisando dados de cinco anos pós-intervenção em 358 pacientes com EAo grave e inoperáveis, constataram que

\footnotetext{
5 Smith et al. N Engl J Med. 2011;364:2187-98; Kodali et al. N Engl J Med. 2012;366:1686-95; Mack et al. Lancet. 2015;385:2477-84.

6 Adams et al. N Engl J Med. 2014;370:1790-8; Reardon et al. J Am Coll Cardiol. 2015;66:113-21; Deeb et al. Eur Heart J. 2016;67:2565-74.

7 Thyregod et al. J Am Coll Cardiol. 2015;65:2184-94.

8 Leon et al. N Engl J Med. 2016;374:1609-20.
}

\begin{tabular}{|c|c|c|c|c|}
\hline Ensaio & TAVI & AVR & Morte por todas as causas & HR (IC95\%) p \\
\hline Partner $1 \mathrm{~A}$ & $116 / 348$ & $114 / 351$ & - & $0,90(0,71,1,15)$ \\
\hline US CoreValve & $85 / 391$ & $99 / 359$ & & $0,79(0,61,1,01)$ \\
\hline Notion & $11 / 145$ & $14 / 135$ & & $0,72(0,33,1,59)$ \\
\hline Partner 2A & $166 / 1011$ & $170 / 1021$ & & $0,92(0,74,1,13)$ \\
\hline \multicolumn{3}{|c|}{ Overall (Heterogeneity $r^{2}<0,001, P=0,755$ ) } & i) & $0,87(0,76,0,99) 0,038$ \\
\hline & & 0,2 & 0,5 & 5 \\
\hline
\end{tabular}

Figura 3. Forest plot para o desfecho de morte por todas as causas na comparação entre TAVI e AVR por ensaios clínicos randomizados (Siontis et al., 2016). 
o risco de morte por todas as causas foi de $71,8 \%$ no grupo TAVI (28,2\% de sobrevida) e de $93,6 \%$ no grupo do tratamento conservador (6,4\% de sobrevida), o que resulta em um hazard ratio (HR) de 0,50 (IC 95\% 0,39-0,65; $p<0,0001$ ), e porcentagem significativamente maior de pacientes do grupo tratado por TAVI (86\%) evoluiu para as classes I e II da NYHA em relação ao tratamento conservador (60\%) (Kapadia et al., 2015). Tais dados mostram que a substituição do tratamento conservador por TAVI obtém não apenas ganhos de sobrevida, mas também de qualidade de vida.

\section{Diferentes opções de biopróteses para TAVI}

Desde o lançamento da primeira bioprótese para TAVI pela empresa Edwards ${ }^{9}$, outros fabricantes vêm apresentando suas próprias válvulas, assim como algumas já lançadas foram melhoradas, buscando resolver algumas dificuldades observadas com os primeiros modelos. Disso resultaram não apenas diferentes marcas, mas também três gerações de biopróteses para TAVI, com diferenças estruturais, resultando em melhores desfechos, conforme foi sendo observado nos vários estudos publicados ao longo do tempo. É importante conhecer tais diferenças, já que elas podem implicar maiores ou menores custos de tratamento e impactar nas decisões médicas, éticas e até econômicas. Um exemplo disso foi publicado por Arnold et al. (2014), que encontraram elevações de até 25\% nos custos hospitalares decorrentes de complicações associadas a diferentes dispositivos para TAVI (Arnold et al., 2014).

Em termos gerais, as biopróteses para TAVI são divididas em: i. válvulas expansíveis por balão, representadas principalmente pela linha Sapien ${ }^{\oplus}$ (Sapien ${ }^{\oplus}$, Sapien $X T^{\oplus}$ e Sapien $3^{\circledR}$, da Edwards); e ii. válvulas autoexpansíveis, representadas principalmente pelas marcas CoreValve ${ }^{\circledast}$ e Evolut $\mathrm{R}^{\circledast}$ (Medtronic $^{10}$ ). Além dessas duas marcas de maior representatividade no mercado, ainda podem ser citadas as marcas Lotus $^{\circledast}$ (Bos- $^{-}$

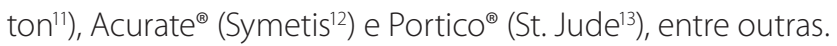

Os desfechos de maior importância na análise crítica do desempenho de válvulas TAVI são a mortalidade e as morbidades (principalmente cardiovascular e renal), avaliados durante a internação primária e nos prazos de 30 dias e 1 ano, que em alguns estudos se estendeu para períodos maiores (três e cinco anos, ou mais). Além desses, há outros que implicam em riscos aumentados de morbimortalidade (sangramento, vazamento paravalvular e regurgitação) e outros que servem como indicadores do desempenho global da válvu-

9 Edwards Lifesciences LLC - Irvine, CA, EUA.

10 Medtronic plc - Dublin, Irlanda.

11 Boston Scientific Corporation - Marlborough, MA, EUA.

12 Symetis AS - Ecublens, Suíça.

13 St. Jude Medical - St. Paul, MI, EUA (recentemente adquirida pela Abbott Laboratories, EUA). la, tais como o tempo para a implantação, a necessidade de substituição do implante, o tempo de hospitalização, a taxa de reospitalizações e o consumo de recursos, entre outros. Por último, e não menos importante, há ainda a variação dos escores de qualidade de vida associada à saúde.

Para sumarizar as diferenças entre os dispositivos para TAVI, algumas comparações serão apresentadas na forma dos grupos a seguir: i. válvulas expansíveis por balão versus válvulas autoexpansíveis, ii. válvulas para TAVI de diferentes gerações e iii. válvulas para TAVI de diferentes fabricantes.

\section{Válvulas expansíveis por balão versus válvulas autoexpansíveis}

Uma comparação entre uma válvula expansível por balão (Sapien 3 ${ }^{\circledR}$, Edwards) e uma válvula autoexpansível (CoreValve ${ }^{\oplus}$, Medtronic) foi realizada por Klein \& Boleckova (2017), por meio da avaliação retrospectiva de 195 casos tratados no University Heart Centre Freiburg (Alemanha), entre os anos 2010 e 2014. O objetivo do estudo foi avaliar se, para pacientes inoperáveis com EAo grave, o consumo de recursos de saúde associados com o uso de TAVI poderiam ser reduzidos pela substituição de válvulas autoexpansíveis (VAE) por válvulas expansíveis por balão (VEB). Para o desfecho de morte por qualquer causa em 30 dias, a diferença entre as duas tecnologias não mostrou significância estatística (3\% para VEB e 8,4\% para $V A E, p=0,101)$. No entanto, vários parâmetros que têm potencial interesse na avaliação de custos mostraram vantagens para as VEB, conforme pode ser observado na Tabela 1, na qual são apresentados todos os parâmetros com diferenças estatisticamente significativas (Klein \& Boleckova, 2017).

$\mathrm{Na}$ conclusão dessa publicação, os autores desse estudo afirmam que, de forma geral, a substituição de válvulas au-

Tabela 1. Análise comparativa do consumo de recursos entre válvulas expansíveis por balão e autoexpansíveis em um hospital da Alemanha (Klein \& Boleckova, 2017)

\begin{tabular}{lccc}
\hline Parâmetro & VEB & VAE & P \\
\hline $\begin{array}{l}\text { Tempo para o } \\
\text { procedimento (min) }\end{array}$ & $88,4 \pm 39,7$ & $115 \pm 42,9$ & $<0,001$ \\
\hline $\begin{array}{l}\text { Gasto de tempo por profissional envolvido (min) } \\
\quad \begin{array}{l}\text { Médicos } \\
\text { Enfermeiros }\end{array}\end{array}$ & $273 \pm 146 \pm 121$ & $360 \pm 188$ & $<0,001$ \\
\hline $\begin{array}{l}\text { Tempo de uso da } \\
\text { fluoroscopia (min) }\end{array}$ & $15,5 \pm 8,7$ & $19,4 \pm 11,2$ & 0,008 \\
\hline $\begin{array}{l}\text { Volume de contraste } \\
\text { utilizado (mL) }\end{array}$ & $285 \pm 80$ & $180 \pm 125$ & 0,002 \\
\hline $\begin{array}{l}\text { Permanência em UTI (dias) } \\
\text { 2,51 }\end{array}$ & 8,04 & $3,56 \pm 2,86$ & 0,004 \\
\hline $\begin{array}{l}\text { Tempo de } \\
\text { internação (dias) }\end{array}$ & $8,69 \pm 3,81$ & $11,11 \pm 5,53$ & 0,001 \\
\hline
\end{tabular}

VEB: válvula expansível por balão; VAE: válvula autoexpansível.

Todos os resultados são apresentados na forma de valor médio e desvio-padrão. 
toexpansíveis por válvulas expansíveis por balão reduziu os custos e aumentou a eficiência do tratamento, sem prejudicar a segurança dos pacientes (Klein \& Boleckova, 2017).

Abdel-Wahab et al. (2014) empreenderam um estudo denominado CHOICE, que comparou dispositivos para TAVI expansíveis por balão versus autoexpansíveis. Pacientes com EAo grave de alto risco foram randomizados para receber um implante de válvula expansível por balão Sapien $X^{\circ} T^{\circ}(121$ pacientes) ou de válvula autoexpansível CoreValve ${ }^{\circledast}(120$ pacientes). A implantação bem-sucedida da válvula ocorreu em $95,9 \%$ dos pacientes no grupo VEB versus $77,5 \%$ no grupo VAE $(p<0,001)$. A necessidade de implantar uma segunda válvula ocorreu em $0,8 \%$ para VEB e em 5,8\% de VAE $(p<0,03)$. A necessidade de implantar um marca-passo permanente foi de $17,3 \%$ no grupo VEB e de $37,6 \%$ no grupo VAE $(p=0,001)$. As taxas de sangramento, lesão vascular e morte por causas cardiovasculares não mostraram diferenças estatisticamente significativas. Os autores concluíram que em pacientes com EAo de alto risco, o uso de válvulas expansíveis por balão resultou em melhores desfechos procedurais em relação à válvula autoexpansível (Abdel-Wahab et al., 2014).

\section{Aspectos econômicos do uso de TAVI}

Além dos custos dos insumos, os custos englobados em uma análise econômica de tecnologias em saúde devem considerar a eficiência dos gastos (custos em relação aos resultados alcançados), o consumo de recursos (tempo de cirurgia, número de profissionais, necessidade de materiais complementares, utilização de equipamentos e medicamentos) e o impacto das complicações. As complicações são, frequentemente, um elemento de custo que pode acarretar forte impacto nos resultados econômicos, seja porque cada evento adverso tem custo elevado, seja porque as adversidades ocorrem com muita frequência, ou ambos os fenômenos ao mesmo tempo.
Embora todos reconheçam esse fato, o modelo de remuneração praticado em uma base de fee-for-service não incentiva a busca da otimização clínica e as operadoras de planos de saúde não têm bons registros da frequência e dos custos que os eventos adversos vêm causando. Isso gera a busca da redução dos valores gastos com a compra de insumos, em vez de buscar a redução dos custos da saúde.

Particularmente para quem paga as contas dos serviços de saúde prestados, é necessário compreender que a qualidade total, envolvendo as melhores técnicas, os melhores processos e os melhores produtos não implica, necessariamente, estar "gastando mais", já que o investimento na seleção de itens e processos que tendem a gerar menores taxas de complicações tem o potencial de aumentar os custos reais e os de oportunidade (termo usado em economia para indicar o custo de algo em termos de uma oportunidade renunciada). Tal raciocínio, descrito de maneira simplista, significa que não adiantará gastar menos na compra de um dispositivo barato se, depois de implantado, ele acarretar complicações cuja proporção leve os custos totais a superarem aqueles esperados com o produto que, a princípio, parecia mais caro.

Várias publicações apontam que os diferentes dispositivos para TAVI mostram variações em fatores que implicam maiores ou menores custos, tal como é apresentado na Tabela 2.

Com base na incidência de complicações periprocedurais oriundas de estudos clínicos (Tabela 3) e os custos brasileiros (hospitais particulares), realizou-se uma modelagem matemática de padrão determinístico para obter o impacto econômico e estimar o incremento de gastos que pode ocorrer pela utilização de diferentes dispositivos para TAVl; esse tipo de modelo pode ser adaptado com os custos próprios de cada país, embora a frequência das complicações seja obtida dos estudos clínicos publicados e que não provêm de comparações diretas. Dessa forma, esses valores devem ser entendidos

Tabela 2. Dados de incidência de complicações em 30 dias após o implante do TAVI, segundo vários estudos

\begin{tabular}{|c|c|c|c|c|c|c|c|c|}
\hline \multirow{2}{*}{$\begin{array}{l}\text { Marca da válvula } \\
\text { para TAVI }\end{array}$} & \multicolumn{2}{|c|}{ VEB, $3^{a}$ geração } & \multicolumn{2}{|c|}{ VEB, $2^{\text {a }}$ geração } & \multicolumn{2}{|c|}{ VAE, $3^{\text {a }}$ geração } & \multicolumn{2}{|c|}{ VAE, $2^{\text {a }}$ geração } \\
\hline & Valor & Ref. & Valor & Ref. & Valor & Ref. & Valor & Ref. \\
\hline Marca-passo & $12,50 \%$ & $\begin{array}{l}\text { Herrmann et al., } \\
2016\end{array}$ & $8,70 \%$ & $\begin{array}{c}\text { Schymik et al., } \\
2015\end{array}$ & $11,7 \%$ & $\begin{array}{l}\text { Manoharan et al., } \\
2015\end{array}$ & $26,00 \%$ & $\begin{array}{c}\text { Barbanti et al., } \\
2016\end{array}$ \\
\hline Infarto do miocárdio & $2,10 \%$ & $\begin{array}{l}\text { Webb et al., } \\
2014\end{array}$ & $0,40 \%$ & $\begin{array}{l}\text { Schymik et al., } \\
2015\end{array}$ & $0,5 \%$ & - & $1,20 \%$ & $\begin{array}{c}\text { Popma et al., } \\
2014\end{array}$ \\
\hline $\begin{array}{l}\text { Complicações } \\
\text { vasculares maiores }\end{array}$ & $4,20 \%$ & $\begin{array}{l}\text { Webb et al., } \\
2014\end{array}$ & $7,90 \%$ & $\begin{array}{l}\text { Schymik et al., } \\
2015\end{array}$ & $8,3 \%$ & $\begin{array}{l}\text { Manoharan et al., } \\
2015\end{array}$ & $8,20 \%$ & $\begin{array}{c}\text { Popma et al., } \\
2014\end{array}$ \\
\hline Falência renal & $1,00 \%$ & $\begin{array}{c}\text { Webb et al., } \\
2014\end{array}$ & $11,90 \%$ & $\begin{array}{l}\text { Schymik et al., } \\
2015\end{array}$ & $1,7 \%$ & $\begin{array}{l}\text { Manoharan et al., } \\
2015\end{array}$ & $11,80 \%$ & $\begin{array}{c}\text { Popma et al., } \\
2014\end{array}$ \\
\hline $\begin{array}{l}\text { Acidente vascular } \\
\text { cerebral }\end{array}$ & $1,00 \%$ & $\begin{array}{l}\text { Herrmann et al., } \\
2016\end{array}$ & $3,40 \%$ & $\begin{array}{l}\text { Schymik et al., } \\
2015\end{array}$ & $0,0 \%$ & $\begin{array}{l}\text { Manoharan et al., } \\
2015\end{array}$ & $4,00 \%$ & $\begin{array}{c}\text { Popma et al., } \\
2014\end{array}$ \\
\hline $\begin{array}{l}\text { Tempo de } \\
\text { hospitalização dias }\end{array}$ & 5 & - & 6 & $\begin{array}{l}\text { Leon et al., } \\
\qquad 2016\end{array}$ & 5 & $\begin{array}{c}\text { Manoharan et al., } \\
2015\end{array}$ & 7 & $\begin{array}{c}\text { Popma et al., } \\
2014\end{array}$ \\
\hline
\end{tabular}


Tabela 3. Custo médio de complicações na saúde suplementar do Brasil

\begin{tabular}{|c|c|c|}
\hline \multirow[b]{2}{*}{ Tipo de complicação } & \multicolumn{2}{|l|}{ Brasil } \\
\hline & US\$ & Ref. \\
\hline Implante de marca-passo & 8.900 & (Orizon, [s.d.]) \\
\hline Infarto do miocárdio & 16.350 & (Ferreira et al., 2016) \\
\hline $\begin{array}{l}\text { Complicações } \\
\text { vasculares maiores }\end{array}$ & 832 & (Orizon, [s.d.]) \\
\hline Insuficiência renal & 15.730 & (de Abreu et al., 2013) \\
\hline Acidente vascular cerebral & 1.389 & (Nishikawa et al., 2013) \\
\hline Diárias de hospitalização & 1.000 & (Manfrin et al., 2014) \\
\hline $\begin{array}{l}\text { Acessórios necessários } \\
\text { ao implante* }\end{array}$ & 3.000 & $\begin{array}{l}\text { ("SIMPRO Revista } \\
\text { Hospitalar", 2016) }\end{array}$ \\
\hline
\end{tabular}

* Balão de valvuloplastia aórtica (BAV) para pré-dilatação, kit para pré-dilatação, bainha dilatadora para implante da válvula.

Fonte: Ferreira et al., 2017.

como estimativas, sujeitas à validação no contexto de cada organização. Os resultados dessa modelagem estão descritos na Tabela 4 e resultam no gráfico apresentado na Figura 4.

Uma vez que o conceito da relação entre custo, frequência e preço unitário foi claramente descrito, recomenda-se fortemente que os resultados apresentados na Figura 4 sejam entendidos apenas como um exemplo desse conceito, e não assumido como os verdadeiros custos das complicações, de forma ampla. Tal recomendação está calcada em várias limitações dessa demonstração, das quais se destacam:

- Como todo processo de modelagem matemática, o modelo de custos de complicações se baseia em pressupostos que precisam ser validados no mundo real;

- Os preços utilizados para a elaboração do modelo não refletem os valores praticados em todos os sistemas de saúde ou hospitais;

- Conforme foi mencionado anteriormente, a frequência e a gravidade das complicações tendem a reduzir à medida que os cirurgiões adquirem mais experiência com o uso da tecnologia, numa razão que não é facilmente previsível em uma modelagem matemática.

Obviamente, o preço praticado para cada marca de válvula tem enorme influência no custo total do tratamento. Na presente modelagem não foi incluído o preço de cada válvula em razão de tais valores serem distintos em função de diversos fatores. Assim sendo, a melhor forma de considerar os dados que foram apresentados é reconhecer que qualquer discussão relativa ao custo de utilização de uma válvula para TAVI não pode ser reduzida simplesmente ao preço do dispositivo, isoladamente. Os cálculos aqui apresentados devem servir como base para que se possam adicionar conhecimentos específicos por cada cenário, incluindo a qualidade dos serviços, os preços praticados, as taxas de complicações (que variam até mesmo em função da gravidade do paciente) e outros fatores.

\section{Conclusão}

Este trabalho apresentou as evidências que suportam a eficácia e a segurança da implantação transcateter da válvula aórtica (TAVI), cujos resultados prolongam a qualidade e a expectativa de vida de portadores de EAo grave e sintomática de alto risco ou inoperáveis. Sem o uso da tecnologia TAVI, esses pacientes estão sujeitos à expectativa de vida curta, com baixa qualidade e associada a altos custos, devido às frequentes internações e tratamentos aos quais são submetidos.

Os dados apresentados ao longo desta publicação mostram que, na comparação entre o tratamento conservador (incluindo AVR e valvuloplastia por balão) e o uso de TAVI, a expectativa de sobrevida de portadores de EAo grave aumenta de $55 \%$ para $80 \%$ em um ano e de $6,4 \%$ para $28,2 \%$ em cinco anos, com expressivo aumento da qualidade de vida em pacientes tratados com TAVI. São resultados de magnitude importante para uma população cuja idade média é superior a 80 anos.

Tabela 4. Custos dos recursos consumidos pela utilização de algumas marcas de válvulas TAVI, ponderados pela frequência (valores em US\$)

\begin{tabular}{|c|c|c|c|c|c|c|c|c|c|}
\hline \multirow{2}{*}{$\frac{\text { Item de custo }}{\text { Marca-passo }}$} & \multirow{2}{*}{$\begin{array}{c}\text { Unitário } \\
\$ 8.900,00\end{array}$} & \multicolumn{2}{|c|}{ VEB $3^{a}$ geração } & \multicolumn{2}{|c|}{ VEB 2a geração } & \multicolumn{2}{|c|}{ VAE $3^{\text {a }}$ geração } & \multicolumn{2}{|c|}{ VAE $2^{\text {a }}$ geração } \\
\hline & & $12,5 \%$ & $\$ 1.112,50$ & $8,7 \%$ & $\$ 774,30$ & $11,7 \%$ & $\$ 1.041,30$ & $26,0 \%$ & $\$ 2.314,00$ \\
\hline Infarto do miocárdio & $\$ 16.350,00$ & $2,1 \%$ & $\$ 343,35$ & $0,4 \%$ & $\$ 65,40$ & $0,5 \%$ & $\$ 81,75$ & $1,2 \%$ & $\$ 196,20$ \\
\hline Complicações vasculares maiores & $\$ 832,00$ & $4,2 \%$ & $\$ 34,94$ & $7,9 \%$ & $\$ 65,73$ & $8,3 \%$ & $\$ 69,06$ & $8,2 \%$ & $\$ 68,22$ \\
\hline Falência renal & $\$ 15.730,00$ & $1,0 \%$ & $\$ 157,30$ & $11,9 \%$ & $\$ 1.871,87$ & $1,7 \%$ & $\$ 267,41$ & $11,8 \%$ & $\$ 1.856,14$ \\
\hline Acidente vascular cerebral & $\$ 1.389,00$ & $1,0 \%$ & $\$ 13,89$ & $3,4 \%$ & $\$ 47,23$ & $0,0 \%$ & $\$-$ & $4,0 \%$ & $\$ 55,56$ \\
\hline Custos de internação (diárias) & $\$ 1.000,00$ & 4 & $\$ 4.000,00$ & 6 & $\$ 6.000,00$ & 5 & $\$ 5.000,00$ & 7 & $\$ 7.000,00$ \\
\hline Material adicional (unidades) & $\$ 3.000,00$ & 0 & $\$-$ & 0 & $\$-$ & 1 & $\$ 3.000,00$ & 1,00 & $\$ 3.000,00$ \\
\hline Custo das complicações & & & $\$ 5.661,98$ & & $\$ 8.824,52$ & & $\$ 9.459,52$ & & $\$ 14.490,12$ \\
\hline
\end{tabular}

Referências: Tabelas 2 e 3. 
$\operatorname{R} 16.000,00$

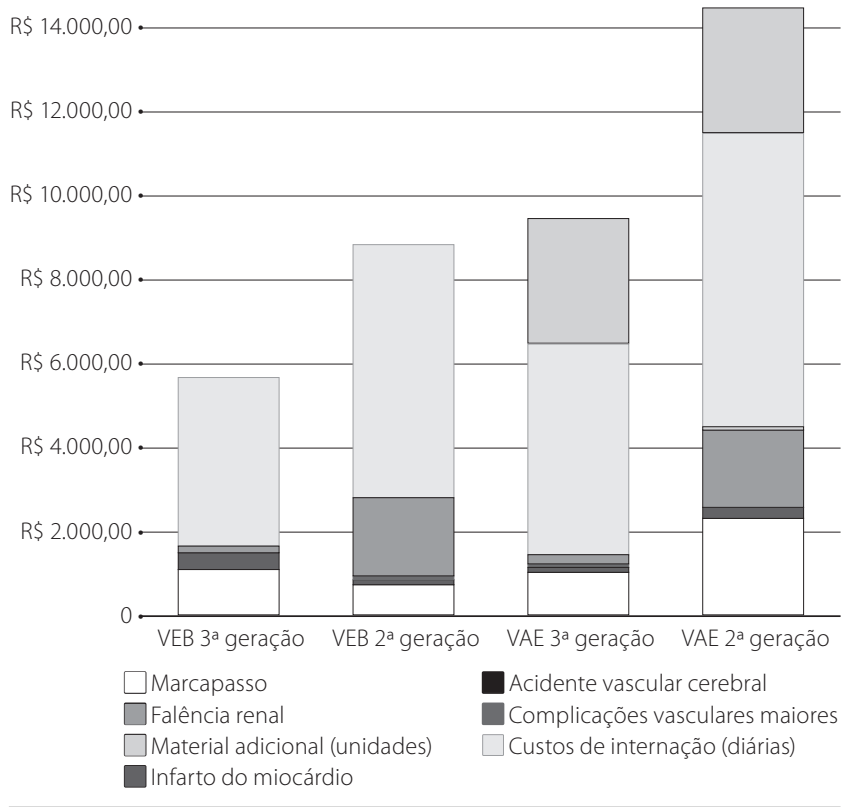

Figura 4. Estimativas de custos para complicações médicas e materiais de implante associados ao uso de algumas marcas de TAVI (no Brasil, valores em US\$). Fonte: Tabela 4.

Também foi observado que as diversas publicações de ensaios clínicos relacionados às diferentes marcas e gerações de dispositivos para TAVI apresentam diferenças nas taxas de eficácia e segurança, que, embora não possam ser usadas em comparações diretas, devem levar a uma análise mais criteriosa do que a simples comparação entre os preços pontuais dos dispositivos. As complicações que ocorrem de forma sistematicamente mais frequente em algumas gerações e marcas dos diferentes modelos de biopróteses certamente causam aumento dos custos, que pode ultrapassar a economia obtida na aquisição. Os resultados de uma modelagem econômica incluídos neste texto mostram o potencial efeito dessas complicações nos custos finais de aplicação, em curto prazo.

Finalizando, é essencial observar que os benefícios médicos e econômicos apresentados neste artigo dependem da adoção de um protocolo adequado de seleção de pacientes e de cuidados, além do treinamento e aquisição de experiência por parte dos cirurgiões.

\section{Referências bibliográficas}

Abdel-Wahab M, Mehilli J, Frerker C, Neumann FJ, Kurz T, Tölg R, et al.; CHOICE investigators. Comparison of balloon-expandable vs selfexpandable valves in patients undergoing transcatheter aortic valve replacement: the CHOICE randomized clinical trial. JAMA. 2014;311(15):1503-14

Amato L, Parmelli E, Vecchi S, Minozzi S, Mitrova Z, Palma R, et al. Protesi valvolari aortiche transcatetere: revisione sistematica della letteratura su efficacia e sicurezza. Recenti Prog Med. 2016;107(1):25-38.
Arnold SV, Lei Y, Reynolds MR, Magnuson EA, Suri RM, Tuzcu EM, et al.; PARTNER Investigators. Costs of periprocedural complications in patients treated with transcatheter aortic valve replacement: results from the Placement of Aortic Transcatheter Valve trial. Circ Cardiovasc Interv. 2014;7(6):829-36.

Barbanti M, Schiltgen M, Verdoliva S, Bosmans J, Bleiziffer S, Gerckens U, et al.; ADVANCE Study Investigators. Three-year outcomes of transcatheter aortic valve implantation in patients with varying levels of surgical risk (from the CoreValve ADVANCE Study). Am J Cardiol. 2016;117(5):820-7.

Brown ML, Pellikka PA, Schaff HV, Scott CG, Mullany CJ, Sundt TM, et al. The benefits of early valve replacement in asymptomatic patients with severe aortic stenosis. J Thorac Cardiovasc Surg. 2008;135(2):308-15.

Clark MA, Arnold SV, Duhay FG, Thompson AK, Keyes MJ, Svensson LG, et al. Five-year clinical and economic outcomes among patients with medically managed severe aortic stenosis: results from a Medicare claims analysis. Circ Cardiovasc Qual Outcomes. 2012;5(5):697-704.

Conitec. Implante por cateter de bioprótese valvar aórtica (TAVI) para o tratamento da estenose valvar aórtica grave em pacientes inoperáveis. Relatório de Recomendação da Comissão Nacional de Incorporação de Tecnologias no SUS - Conitec. Brasília/DF: [s.n.]. Disponível em: http://portalarquivos.saude.gov.br/images/pdf/2014/janeiro/30/TAVIFINAL.pdf.

Ferreira CN, et al. Disease Burden Associated with Aortic Stenosis in Patients uneergoing transcatheter aortic valve replacement, ISPOR 6h Latin America, poster nr. PMD17.

Herrmann HC, Thourani VH, Kodali SK, Makkar RR, Szeto WY, Anwaruddin $\mathrm{S}$, et al. One-year clinical outcomes with SAPIEN 3 transcatheter aortic valve replacement in high-risk and inoperable patients with severe aortic stenosis. Circulation. 2016;134(2):130-40.

Hussain Al, Garratt AM, Brunborg C, Aakhus S, Gullestad L, Pettersen KI. Eliciting patient risk willingness in clinical consultations as a means of improving decision-making of aortic valve replacement. J Am Heart Assoc. 2016;5(3):e002828.

Kapadia SR, Leon MB, Makkar RR, Tuzcu EM, Svensson LG, Kodali S, et al.; PARTNER trial investigators. 5-year outcomes of transcatheter aortic valve replacement compared with standard treatment for patients with inoperable aortic stenosis (PARTNER 1): a randomised controlled trial. Lancet. 2015;385(9986):2485-91.

Klein H, Boleckova J. Resource utilization and procedure-related costs associated with transfemoral transcatheter aortic valve replacement. J Med Econ. 2017;20(6):640-5.

Kodali SK, Doshi D, Leon M. B. 29 - Aortic Valvuloplasty and Transcatheter Aortic Valve Replacement. Cardiovascular Intervention: A Companion to Braunwald's Heart Disease; 2017. p. 451-81.

Leon MB, Smith CR, Mack MJ, Makkar RR, Svensson LG, Kodali SK, et al.; PARTNER 2 Investigators. Transcatheter or Surgical AorticValve Replacement in Intermediate-Risk Patients. N Engl J Med. 2016;374(17):1609-20.

Leon MB, Smith CR, Mack M, Miller DC, Moses JW, Svensson LG, et al.; PARTNER Trial Investigators. Transcatheter aortic-valve implantation for aortic stenosis in patients who cannot undergo surgery. N Engl J Med. 2010;363(17):1597-607.

Manoharan G, Walton AS, Brecker SJ, Pasupati S, Blackman DJ, Qiao H, et al. Treatment of symptomatic severe aortic stenosis with a novel resheathable supra-annular self-expanding transcatheter aortic valve system. JACC Cardiovasc Interv. 2015;8(10):1359-67.

Nashef SA, Roques F, Hammill BG, Peterson ED, Michel P, Grover FL, et al.; EurpSCORE Project Group. Validation of European System for Cardiac Operative Risk Evaluation (EuroSCORE) in North American cardiac surgery. Eur J Cardiothorac Surg. 2002;22(1):101-5. 
Nashef SA, Roques F, Michel P, Gauducheau E, Lemeshow S, Salamon R. European system for cardiac operative risk evaluation (EuroSCORE). Eur J Cardiothorac Surg. 1999;16(1):9-13.

Nishimura RA, Otto CM, Bonow RO, Carabello BA, Erwin JP, Guyton RA, et al. 2014 AHA/ACC Guideline for the Management of Patients With Valvular Heart Disease: A Report of the American College of Cardiology/ American Heart Association Task Force on Practice Guidelines. J Am Coll Cardiol. 2014;63(22).

Osnabrugge RL, Mylotte D, Head SJ, Van Mieghem NM, Nkomo VT, LeReun $C M$, et al. Aortic stenosis in the elderly: disease prevalence and number of candidates for transcatheter aortic valve replacement: a meta-analysis and modeling study. J Am Coll Cardiol. 2013;62(11):1002-12.

Otto CM, Bonow RO. Valvular heart disease. 20th ed. [s.I.] Elsevier Inc.; 2014.

Otto CM, Kumbhani DJ, Alexander KP, Calhoon JH, Desai MY, Kaul S, et al. 2017 ACC Expert Consensus Decision Pathway for Transcatheter Aortic Valve Replacement in the Management of Adults With Aortic Stenosis: A Report of the American College of Cardiology Task Force on Clinical Expert Consensus Documents. J Am Coll Cardiol. 2017;69(10):1313-1346.

Popma JJ, Adams DH, Reardon MJ, Yakubov SJ, Kleiman NS, Heimansohn D, et al.; CoreValve United States Clinical Investigators. Transcatheter aortic valve replacement using a self-expanding bioprosthesis in patients with severe aortic stenosis at extreme risk for surgery. J Am Coll Cardiol. 2014;63(19):1972-81.

Ren X, Lange RA. Aortic stenosis. Disponível em: http://emedicine.medscape. com/article/150638-overview. Acesso em: 23 mar. 2017.

Schwartz JB, Zipes DP. 76 - Cardiovascular Disease in the Elderly. 20th ed. [s.l.] Elsevier Inc.; 2015.

Schymik G, Lefèvre T, Bartorelli AL, Rubino P, Treede H, Walther T, et al. European experience with the second-generation Edwards SAPIEN $\mathrm{XT}$ transcatheter heart valve in patients with severe aortic stenosis: 1-year outcomes from the SOURCE XT Registry. JACC Cardiovasc Interv. 2015;8(5):657-69.
Shroyer AL, Coombs LP, Peterson ED, Eiken MC, DeLong ER, Chen A, et al.; Society of Thoracic Surgeons. The Society of Thoracic Surgeons: 30day operative mortality and morbidity risk models. Ann Thorac Surg. 2003;75(6):1856-64.

Siontis GC, Praz F, Pilgrim T, Mavridis D, Verma S, Salanti G, et al. Transcatheter aortic valve implantation vs. surgical aortic valve replacement for treatment of severe aortic stenosis: a meta-analysis of randomized trials. Eur Heart J. 2016;37(47):3503-12.

Tarasoutchi F, Montera MW, Grinberg M, Barbosa MR, Piñeiro DJ, Sánchez CRM, et al. Diretriz Brasileira de Valvopatias - SBC 2011/I Diretriz Interamericana de Valvopatias - SIAC 2011. Arq Bras Cardiol. 2011;97(5 Supl 1):1-67.

Thaden JJ, Nkomo VT, Enriquez-Sarano M. The global burden of aortic stenosis. Prog Cardiovasc Dis. 2014;56(6):565-71.

Vasques F, Messori A, Lucenteforte E, Biancari F. Immediate and late outcome of patients aged 80 years and older undergoing isolated aortic valve replacement: a systematic review and meta-analysis of 48 studies. Am Heart J. 2012;163(3):477-85.

Webb J, Gerosa G, Lefèvre T, Leipsic J, Spence M, Thomas M, et al. Multicenter evaluation of a next-generation balloon-expandable transcatheter aortic valve. J Am Coll Cardiol. 2014;64(21):2235-43.

Wendler O, Schymik G, Treede H, Baumgartner H, Dumonteil N, Ihlberg L, et al. SOURCE 3 Registry: Design and 30-Day Results of the European Postapproval Registry of the Latest Generation of the SAPIEN 3 Transcatheter Heart Valve. Circulation. 2017;135(12):1123-32. 